\title{
Interactive Modelling and Simulation of Micromirror MEMS Devices
}

\author{
Sarbast Rasheed \\ Department of Engineering, American University of Iraq, Sulaimani (AUIS), Sulaimani, Iraq; \\ *sarbast.rasheed@auis.edu.krd
}

SNE 28(3), 2018, 137 - 140, DOI: 10.11128/sne.28.sn.10438

Received: Sept. 15, 2016 (Selected EUROSIM Congress 2016 Postconf. Publ.), Revised August 25, Accepted: Sept. 5, 2018 SNE - Simulation Notes Europe, ARGESIM Publisher Vienna, ISSN Print 2305-9974, Online 2306-0271, www.sne-journal.org

Abstract. This paper presents an interactive software package for the modelling and simulation of microelectromechanical system (MEMS) devices utilizing the MATLAB high-level programming language and its interactive environment. The package provides a dynamic analysis and frequency responses of a 1-D torsional micromirror electrostatically actuated with staggered vertical combdrives. Applying a frequency sweep to the micromirror equation, the torsional mode natural frequency may be estimated. The developed package consists of several graphical user interfaces used for modeling MEMS devices. The software package is useful for computing the forces and torques for different micromirror geometries.

\section{Introduction}

Behaviour of microelectromechanical system (MEMS) is often very sensitive to geometry and applied voltages; and their responses could be very non-linear. Therefore, mathematical modelling becomes a very important technique for gaining physical understanding of these systems. To predict the behaviour of MEMS, the shape and material properties are used as inputs with a goal to develop models to enable better understanding of MEMS performance and help in the design and fabrication of MEMS to achieve optimum performance. For this purpose, an interactive system is developed for modeling micromirror MEMS devices. The developed system exhibits a style of control and works as an interactive environment for modeling MEMS devices. It provides users with a full control of the process of predicting the behaviour of micromirror MEMS devices.
Modeling the performance of MEMS devices involves computing the deformations and stresses on structures subjected to electric fields, and hence electrostatic forces. The change of shape changes the capacitance [1] of these structures and this in turn changes the forces on them. The final configuration must satisfy both the electric field as well as the mechanics of equations.

The dynamic analysis of MEMS devices is complicated by the fact that there are two physical domains: electrical and mechanical with nonlinear coupling between them. In this paper, we are interested in investigating the linear and nonlinear dynamical behavior of a micromirror actuated with single-sided and doublesided vertical combdrives [2] in an interactive fashion. We investigate the electromechanical response of a scanning micromirror in the linear and nonlinear regions of the electrostatic torque induced by its actuators. The nonlinear behavior of the frequency response with small and large scanning angles is examined using upsweep (increasing) frequency mode and down sweep (decreasing) frequency mode.

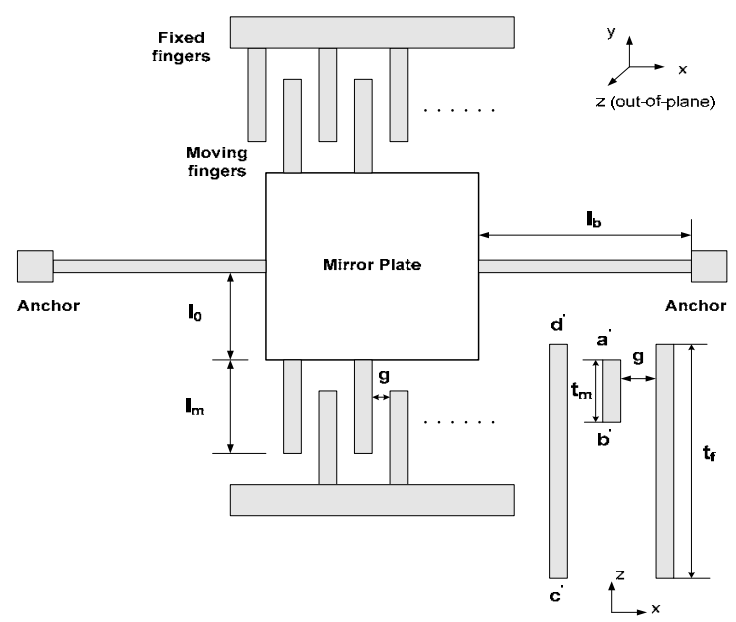

Figure 1. Schematic diagram of a micromirror actuated with double-sided staggered vertical combdrives. 


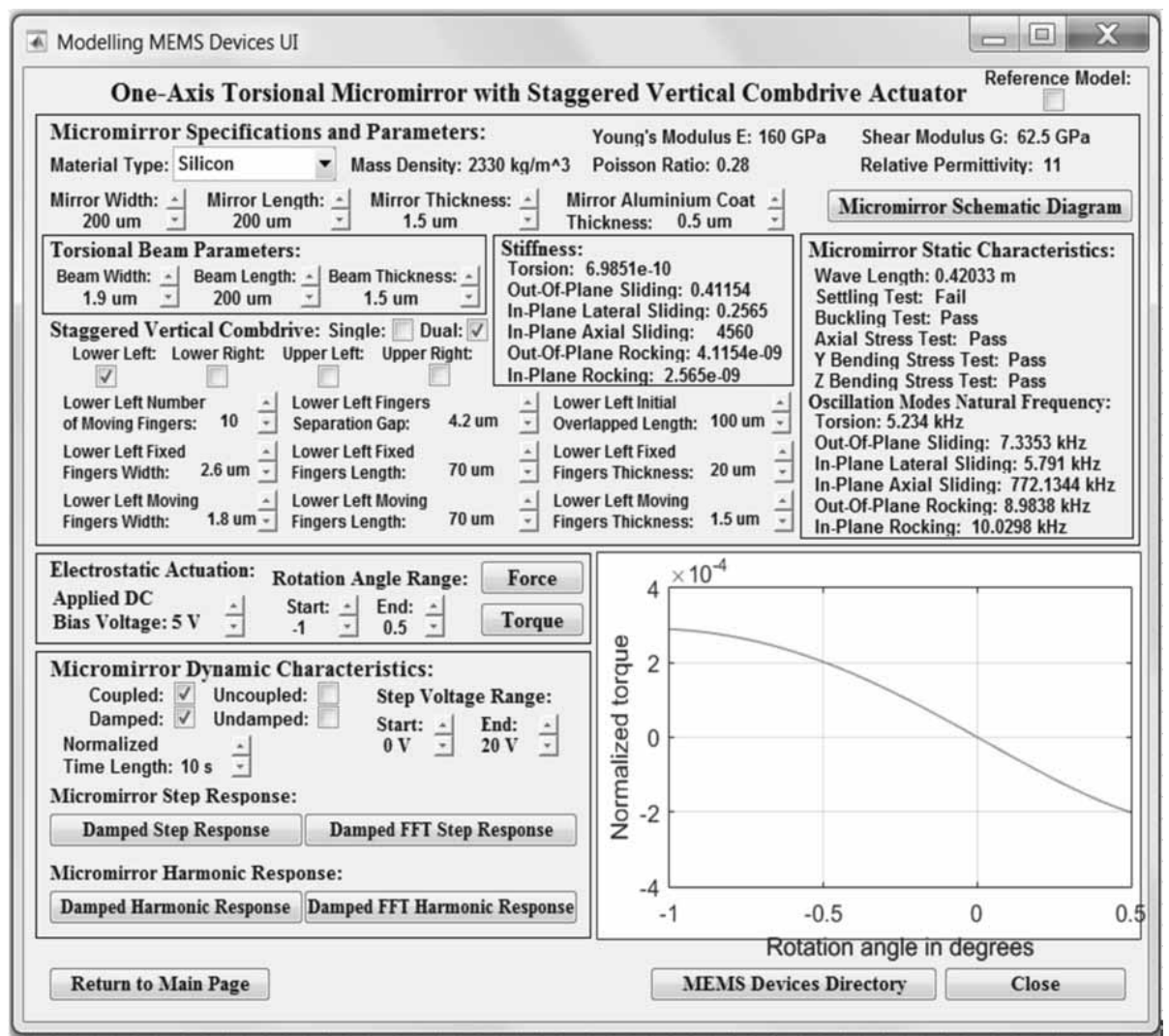

Figure 2. The MATLAB user interface for modeling a scanning micromirror with dual staggered vertical combdrive actuator.

\section{Staggered Vertical Combdrive}

Out-of-plane rotational scanning may be achieved within micromirrors using staggered vertical combdrive (SVC) actuators as shown in Figure 1. SVC requires a vertical offset between the moving fingers (rotor) and the fixed fingers (stator) for out-of-plane rotation. An electrostatic field between these two sets of combs will produce an out-of-plane force and a torque around the torsional axis of the mirror as a result of the fringing field applied to the movable fingers.

The equation of motion of the micromirror rotation around the $\mathrm{x}$-axis using a lumped-mass model can be written as:

$$
I_{m} \frac{d^{2} \theta}{d t^{2}}+C_{d} \frac{d \theta}{d t}+K_{s} \theta=T_{e}(\theta)
$$

where $I_{m}$ is the mass moment of inertia of the micromirror; $C_{d}$ is the damping coefficient; $K_{s}$ is the torsion stiffness; $\theta$ is the tilt angle of the micromirror; and $T_{e}$ is the torque contributed by the electrostatic force between the fixed fingers and movable fingers of the SVC actuator.

\section{Design of SVC Actuator}

The angular motion induced by the SVC actuator is due to the generated torque, $T_{e}$, given by [3], [4]:

$$
\begin{aligned}
& T_{e}=2 N_{f} \frac{\varepsilon_{0} \pi^{2} V^{2}}{4 g} \int_{l_{0}}^{l_{0}+l_{m}} \frac{1}{(a-b)}\left(a \frac{b-d}{a-d}-b \frac{a-c}{b-c}\right) \\
& \frac{z d z}{[F(\partial(u=0), q)+F(\kappa(u=0), q)]^{2}} .
\end{aligned}
$$

where $V$ is the voltage difference between the combs; $\varepsilon_{0}$ is the free-space permittivity, $N_{f}$ is the number of movable fingers, $g$ is the gap between the fingers, $l_{0}$ is the distance from the axis of rotation to the root of the moving fingers, $l_{m}$ is the distance from the root to the tip of the moving finger, $F$ denotes the incomplete elliptic function of the first kind, and the dimensionless parameters $\partial, \kappa$, and $q$ are defined as follows: 
$q=\sqrt{\frac{(b-c)(a-d)}{(a-c)(b-d)}}, \quad \partial=\sin ^{-1} \sqrt{\frac{(b-d)(u-c)}{(b-c)(u-d)}}$,

$\kappa=\sin ^{-1} \sqrt{\frac{(a-c)(b-u)}{(b-c)(a-u)}}$.

Assuming the width of the comb fingers to be infinitely small, the parameters $a, b, c, d$ represent the conformal mapping of the $\mathrm{x}-\mathrm{z}$ plane boundaries of a combdrive unit comprised of half a movable finger $a^{\prime}, b^{\prime}$ and half a fixed finger $c^{\prime}, d^{\prime}$ into the w-plane using the transformation function $w=e^{\pi z / g}$, where $w=u+j v$.

The plot inside the user interface of Figure 2 shows the normalized torque per unit combdrive as a function of $\theta$, where $T_{e}=2 N_{f} \cdot T_{\text {unit }}$. For small angular displacement, $T_{\text {unit }}$ can be approximated by a fourth-order polynomial in $\theta$ such that:

$$
T_{\text {unit }} \approx \frac{\varepsilon_{0} \pi^{2} V^{2}}{4}\left(p_{4} \theta^{4}+p_{3} \theta^{3}+p_{2} \theta^{2}+p_{1} \theta+p_{0}\right)
$$

where $p_{4}, p_{3}, p_{2}, p_{1}, p_{0}$ are the coefficients of the polynomial fit. Then the nonlinear equation of motion (1) can be solved numerically to determine the micromirror response.

\section{Interactive Micromirror Dynamic Analysis}

The goal of the interactive dynamic analysis is to develop a convenient model for estimating the fundamental (first) natural frequency (the torsional mode). The dynamic modelling in this paper is based on geometric dimensions of a micromirror presented below:

$$
\begin{array}{ll}
\text { Torsional bars } & \text { length } l_{b}=200 \mu \mathrm{m} \\
& \text { width } w_{b}=1.9 \mu \mathrm{m} \\
& \text { thickness } t_{b}=1.5 \mu \mathrm{m} \\
\text { Micromirror } & \text { length } l_{r}=200 \mu \mathrm{m} \\
& \text { width } w_{r}=200 \mu \mathrm{m} \\
& \text { thickness } t_{r}=1.5 \mu \mathrm{m} \\
\text { Movable fingers } & \text { length } l_{m}=70 \mu \mathrm{m} \\
& \text { width } w_{m}=1.8 \mu \mathrm{m} \\
& \text { thickness } t_{m}=1.5 \mu \mathrm{m}
\end{array}
$$

Fixed fingers length $l_{f}=70 \mu \mathrm{m}$

width $w_{f}=2.6 \mu \mathrm{m}$

thickness $t_{f}=20 \mu \mathrm{m}$

Separation gap between fingers $g=4.2 \mu \mathrm{m}$

Number of moving fingers $N_{f}=10$.

The above micromirror and comb-drive geometric dimensions; and the material properties have been specified in the control objects of the user interface shown in Figure 2 for a double-sided vertical comb-drive. Figure 2 also shows the plot of the unit torque $T_{\text {unit }}$ versus tilt angle $\theta$ varied from -1.0 to 0.5 degrees.

To determine the micromirror response, shown in Figure 4 and Figure 5, a 5 volt amplitude sinusoidal excitation voltage with upsweep frequency ranges $\Omega$ is applied for 50 seconds, as shown in Figure 3, starting from $4200 \mathrm{~Hz}$ and then solving the equation of motion numerically. From Figure 4, we can estimate the torsional mode natural frequency to be $4340 \mathrm{~Hz}$.

For a single-sided vertical comb-drive, Figure 6 shows the specification of the micromirror and combdrive geometric dimensions; and the material properties in the control objects of the user interface. Figure 6 also shows the plot of the unit torque $T_{\text {unit }}$ versus tilt angle $\theta$ varied from -2.0 to 3.0 degrees.

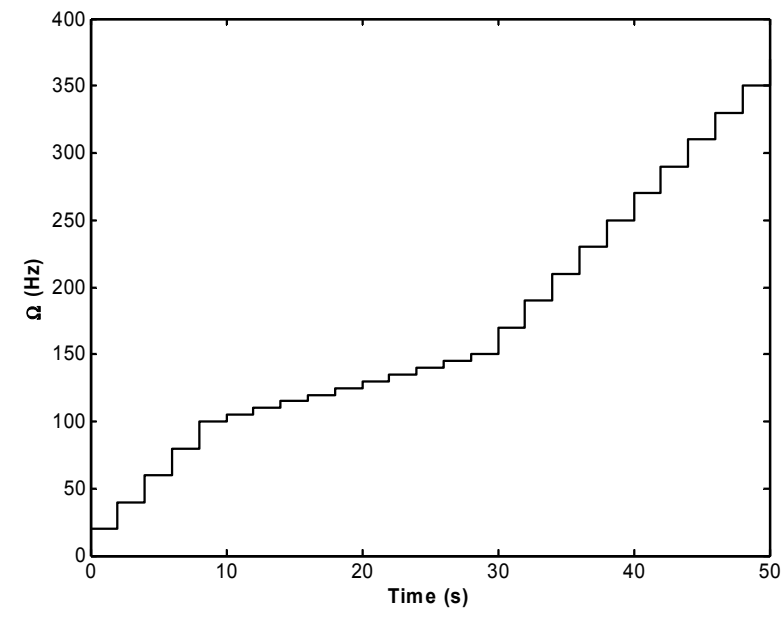

Figure 3. Upsweep frequency $\Omega$ function for $50 \mathrm{~s}$. 


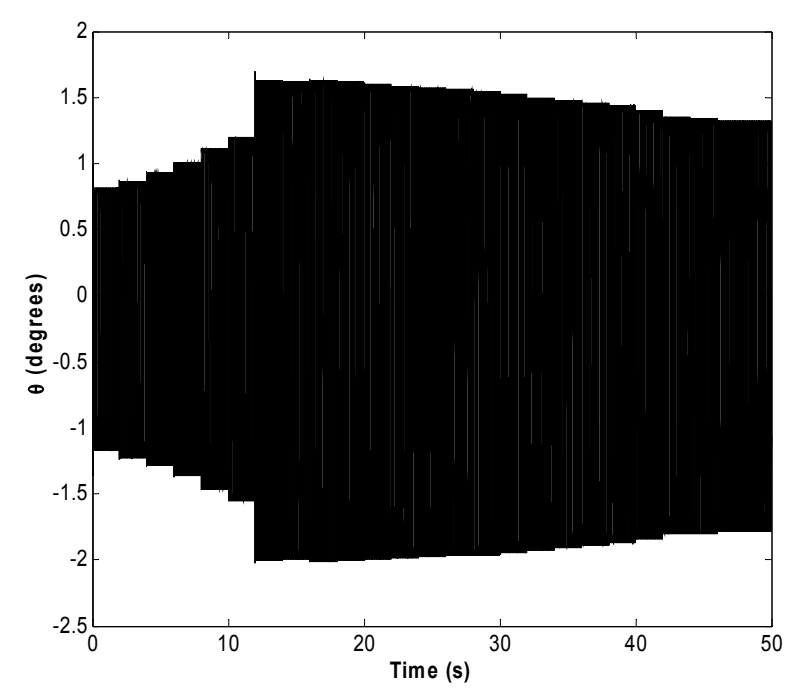

Figure 4. Micromirror response due to sinusoidal excitation voltage with upsweep frequency ranges.

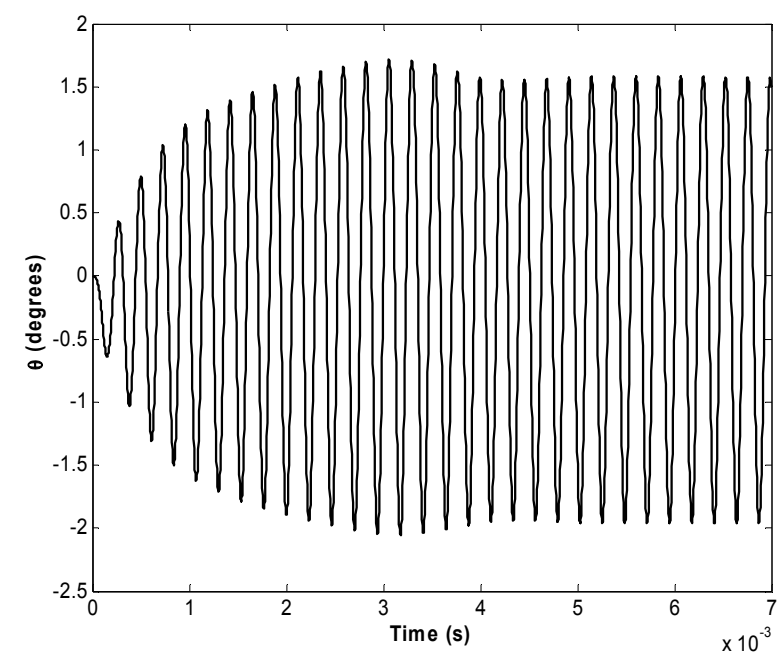

Figure 5. Micromirror response for $7 \mathrm{~ms}$ driven at the estimated natural frequency $4340 \mathrm{~Hz}$.

\section{Conclusion}

An automated method for the modeling and simulation of MEMS devices in the form of an interactive modeling software package had been constructed. The package provides users with a full control of the process and allows them to experiment with different configurations and parameters that can be selected and changed using the displayed control objects of the user interface. The developed environment is convenient and easy to use and change as it involves multiple runs designed to investigate the effects of different system parameters, initial conditions, and model changes.

\section{References}

[1] Chang CM, Wang SY, Chen R, Yeh JA, Hou MT. "A comb-drive actuator driven by capacitively-coupledpower," Sensors, vol. 12, no. 8, pp. 10881-10889, 2012.

[2] Rasheed S, Yeow J, McPhee J. "Dynamic analysis of micromirrors with staggered vertical combdrives actuation,"in Proceedings of the 21st Canadian Congress of Applied Mechanics (CANCAM) 2007 Conference, Ryerson University, Toronto, Canada, June 3-7, 2007, pp. 585-586.

[3] Yeh JLA, Hui CY, Tien NC. "Electrostatic model for an asymmetric combdrive," IEEE Journal of Microelectromechanical Systems, vol. 9, no. 1, pp. 126-135, March 2000.

[4] Hui CY, Yeh JLA, Tien NC. "Calculation of electrostatic forces and torques in MEMS using path-independent integrals," Journal of Micromechanics and Microengineering, vol. 10, no. 3, pp. 477-482, 2000. 